\title{
Cosmesis after early stage breast cancer treatment with surgery and radiation therapy: experience of patients treated in a Chilean radiotherapy centre
}

\author{
Lorena Vargas ${ }^{1,2}$, Sebastián Solé ${ }^{3}$ and Claudio Vicente Solée ${ }^{3,4}$ \\ ${ }^{1}$ Radiotherapy Resident, Clínica IRAM, Santiago 7630370, Chile \\ ${ }^{2}$ Faculty of Medicine, Universidad Diego Portales, Santiago 8370068, Chile \\ ${ }^{3}$ Radiation Oncology, Clínica IRAM, Santiago 7630370, Chile \\ ${ }^{4}$ Statistical analysis, Clínica IRAM, Santiago 7630370, Chile
}

Correspondence to: Lorena Vargas. Email: levargas@miuandes.cl

\section{Abstract}

Aim: To analyse the overall cosmetic outcome according to patient self-assessment in relation to the fractionation received.

Materials and methods: A questionnaire, drawn up on the basis of subjective rating scales of cosmesis and of acute and late toxicity RTOG/EORTC, EORTC QLQ-C30 (v3) and LENT SOMA, was applied to patients with early-stage breast cancer who received radiotherapy with tangential fields between June 2014 and July 2015. Self-perception of cosmesis, changes in the treated breast, pain and fractionation used (hypofractionation (HF) 42.56 Gy in 16 fractions or conventional fractionation (CF) 50 Gy in 25 fractions) were evaluated.

Results: Three hundred and fifty-two questionnaires were obtained. The median age was 58 years. $45 \%$ of patients reported 'excellent' cosmesis, 53\% 'good', and $2 \%$ 'poor' cosmesis. Cosmesis was considered 'excellent/good' by $98 \%$ of patients. No statistically significant difference was found in overall cosmesis according to fractionation received $(p=0.6)$.

The most frequent alteration was 'difference between both breasts' (77\%), and 48\% reported change in normal breast colour.

Fifteen percent of patients who are younger than 58 years reported a change of normal breast colour affecting cosmesis compared to $9 \%$ of patients older than 58 years $(p=0.04)$.

Complications affecting breast cosmesis were reported by $9 \%$ of patients with stages I-II compared with $2 \%$ with cancer in situ (DCIS) $(p=0.04) ; 14 \%$ in stages I-II referred colour change affecting cosmesis compared to $6 \%$ of those with DCIS $(p=0.03)$.

Ninety-four percent of patients stated that they would accept treatment again.

Published: 21/03/2018

Received: 03/10/2017

ecancer 2018, 12:819 https://doi.org/10.3332/ecancer.2018.819

Copyright: (C) the authors; licensee ecancermedicalscience. This is an Open Access article distributed under the terms of the Creative Commons Attribution License (http://creativecommons.org/licenses/by/3.0), which permits unrestricted use, distribution, and reproduction in any medium, provided the original work is properly cited. 
Conclusions: No difference in cosmetic results was found between HF and CF in our patients. Great satisfaction regarding cosmetic outcome of cancer treatment was reported, given by $98 \%$ of excellent/good cosmesis, and $94 \%$ of patients who would receive treatment again.

Keywords: breast cancer, cosmesis, hypofractionation

\section{Background}

Adjuvant radiation therapy after conservative breast surgery represents the standard treatment to improve loco-regional control and survival [1]. Since several studies have demonstrated noninferiority in terms of disease control, survival and toxicity, it is now possible to offer both conventional fractionation radiotherapy (CFRT) and hypofractionated schedules, as they also have the advantage of lower total treatment time [2-5].

The treatment of breast cancer can result in discomfort such as pain and alterations in cosmesis by disturbances in the volume of the breast, scars and skin changes. These changes result from both surgery and adjuvant treatments. Radiation therapy can cause both acute and chronic damages, while the effects of the surgery are usually more immediate [6]. Radiation toxicity can manifest by telangiectasia, oedema and fibrosis of subcutaneous tissue, leading to loss of volume and retraction of the breast. Fibrosis can manifest as induration, to which oedema may also contribute [2].

The study by Whelan et al [2] showed no differences in toxicity to skin or subcutaneous tissues at 10 years of follow-up, showing an incidence of late grade 2 toxicity of $5 \%$ and $6.4 \%$ with $\mathrm{CF}$ and hypofractionation (HF), respectively, and $2.7 \%$ and $2.5 \%$ grade 3 , respectively. Also, the START A and B studies showed lower toxicity with hypofractionated schedule, reflected in reduced shrinkage, induration, telangiectasias and breast oedema [4,5]. Moreover, recent results of a randomized study evaluating acute and 6 months toxicity, and quality of life in patients treated with CFRT compared with HF have been published. The latter group had less fatigue, itching, dermatitis and hyperpigmentation according to the evaluation made by the treating physician [7].

The aim of early-stage breast cancer treatment is not only the oncologic outcome but also the result of cosmesis, which is also a measure of quality of life [8]. Cosmesis and toxicity are usually reported by a physician or a third observer [9, 10]; however, patient self-assessment is of particular interest as well.

The aim of this study is to analyze the overall cosmetic outcome of early stage breast cancer after RT treatment by a patient self-assessment according to the fractionation received.

\section{Materials and methods}

A questionnaire was drawn up on the basis of subjective rating scales of cosmesis $[4,5,8,9]$ also considering the scales of acute and late toxicity RTOG/EORTC, EORTC QLQ-C30 (version 3) and LENT SOMA. From June 2014 to July 2015, this questionnaire was applied to patients diagnosed with early-stage breast cancer treated with conservative surgery, who had received or were to receive adjuvant RT only with tangential fields, with no fields planned to nodal areas, in a Chilean radiotherapy institution. The survey could be applied before the start of RT, at the end of the treatment and/or in follow-up visits by the treating physician or resident. There was no specific interval schedule for follow-up visits.

Patients were asked to respond according to their own perception if they found differences between the two breasts, if they had any alteration of the form of the treated breast, induration, scar complication, alteration on nipple-areola complex and change in normal colour of the breast. Patients were also asked about the presence of pain, and general aesthetic outcome assessment. Age, stage at diagnosis, fractionation used (HF $42.56 \mathrm{~Gy}$ in 16 fractions [2] or CF of $50 \mathrm{~Gy}$ in 25 fractions), the use of a surgical bed boost and field in field technique were also recorded. Figure 1 shows the questionnaire used for assessment. All treatment decisions were made in a multidisciplinary tumour board and all patients signed the written informed consent. 


\begin{tabular}{|c|c|c|c|}
\hline NAME: & & AGE: & \\
\hline & None & $\begin{array}{l}\text { Yes, but it doesn't } \\
\text { affect me }\end{array}$ & $\begin{array}{c}\text { Yes, and it affects } \\
\text { me }\end{array}$ \\
\hline \multicolumn{4}{|l|}{ Difference between both breasts } \\
\hline \multicolumn{4}{|l|}{ Shape alteration (deformity) } \\
\hline \multicolumn{4}{|l|}{ Breast induration } \\
\hline \multicolumn{4}{|l|}{ Scar alteration or complication } \\
\hline \multicolumn{4}{|l|}{ Nipple/areola alteration } \\
\hline \multicolumn{4}{|l|}{ Change in normal color } \\
\hline \multicolumn{4}{|l|}{ Others } \\
\hline Pain & Permanent & Occasional & No \\
\hline General aesthetic result & Excellent & Good & Poor \\
\hline Would you do the treatment again? & Yes & No & \\
\hline Treating physician: & Stage: & & \\
\hline \multicolumn{4}{|l|}{ Date of end of RT: } \\
\hline \multicolumn{4}{|l|}{ Control date: } \\
\hline \multicolumn{4}{|l|}{ TIME OF FOLLOW UP (MONTHS): } \\
\hline Treatment & Field in field & Boost & \\
\hline Hypofractionated & YES No & YES No & \\
\hline
\end{tabular}

Figure 1. Questionnaire.

A descriptive analysis was performed to calculate proportions, frequencies and medians. Chi square and Kruskal Wallis tests were used for analysis of differences between variables when appropriate. Statistical significance was $p<0.05$. Analyses were performed in SPSS v21.

\section{Results}

270 patients completed 352 questionnaires between June 2014 and July 2015, patients included were treated from 1999 to June 2015 , all patients completed treatment as planned. The median age of patients was 58 years old (31-88) and $24 \%$ were younger than 50 years old. Patient, stage at diagnosis and treatment characteristics according to fractionation received are shown in Table 1. 
Table 1. Patient and tumour characteristics.

\begin{tabular}{|c|c|c|}
\hline & $\mathrm{HF}^{*}$ & $\mathrm{CF}^{* *}$ \\
\hline $\mathrm{N}$ & 127 & 143 \\
\hline \multicolumn{3}{|c|}{ Age (years) } \\
\hline $31-50$ & $6(5 \%)$ & $59(41 \%)$ \\
\hline $51-60$ & $44(35 \%)$ & $38(27 \%)$ \\
\hline $61-70$ & $44(35 \%)$ & $32(22 \%)$ \\
\hline$\geq 71$ & $33(26 \%)$ & $14(10 \%)$ \\
\hline \multicolumn{3}{|c|}{ Tumour Stage $\mathrm{e}^{\star \star *}$} \\
\hline 0 & $35(28 \%)$ & $27(19 \%)$ \\
\hline I & $67(53 \%)$ & $82(57 \%)$ \\
\hline II & $25(20 \%)$ & $34(24 \%)$ \\
\hline \multicolumn{3}{|c|}{ Field in field } \\
\hline Yes & $93(73 \%)$ & $43(30 \%)$ \\
\hline No & $34(27 \%)$ & $100(70 \%)$ \\
\hline \multicolumn{3}{|c|}{ Boost $^{* \star * *}$} \\
\hline Yes & $2(<2 \%)$ & $141(99 \%)$ \\
\hline No & $125(98 \%)$ & $2(<2 \%)$ \\
\hline
\end{tabular}

${ }^{*} \mathrm{HF}: 42.56$ Gy in 16 fractions

${ }^{* *} \mathrm{CF}: 50$ Gy in 25 fractions

***Tumour stage according TNM AJCC $7^{\text {a }}$ edition

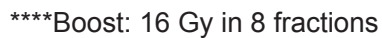

Percentages are rounded to whole numbers

Seventy-one questionnaires were completed at the time of the first visit before radiation treatment, 80 questionnaires at discharge and 201 questionnaires in follow-up visits; 281 questionnaires completed at these two last time points were considered as evaluation of RT treatment effect, 2 were excluded for being incomplete.

Median follow-up was 6 months in both fractionation schedules, and the maximum follow-up was 227 months in a patient treated in 1999. Most questionnaires were completed by patients with less than a year of follow-up (Table 2).

Regarding the overall assessment of cosmesis after RT, 45\% (126/279) reported 'excellent' cosmesis, 53\% (147/279) 'good', and 2\% (6/279) 'poor' cosmesis. Overall cosmesis was considered 'good/excellent' by $98 \%$ (273/279) of patients.

There was no statistical difference in overall cosmesis according to fractionation received $(p=0.6)$ (Figure 2).

No statistically significant difference was found in overall cosmesis regarding the use of boost or field-in-field technique. With regard to the different characteristics interrogated, no statistically significant difference was found in their frequency according to fractionation received.

'Alteration affecting cosmesis' and 'alteration that does not affect cosmesis' were considered together as the 'presence of alteration'. In this analysis, 'difference between both breasts' was described by $77 \%$, followed by 'alteration in shape of the breast' (56\%) and then by 'induration' (53\%). The percentage of patients who reported change in normal breast colour was $48 \%$.

Response distribution for each feature is depicted in Figures 3-5. 
Table 2. Number of questionnaires at different time points.

\begin{tabular}{|l|c|}
\hline Time interval (months) & N of questionnaires \\
\hline Pre-treatment & 71 \\
\hline 0 & 80 \\
\hline $1-4$ & 55 \\
\hline $5-8$ & 46 \\
\hline $9-12$ & 10 \\
\hline $13-16$ & 13 \\
\hline $17-20$ & 17 \\
\hline $21-27$ & 14 \\
\hline$\geq 28$ & 46 \\
\hline
\end{tabular}

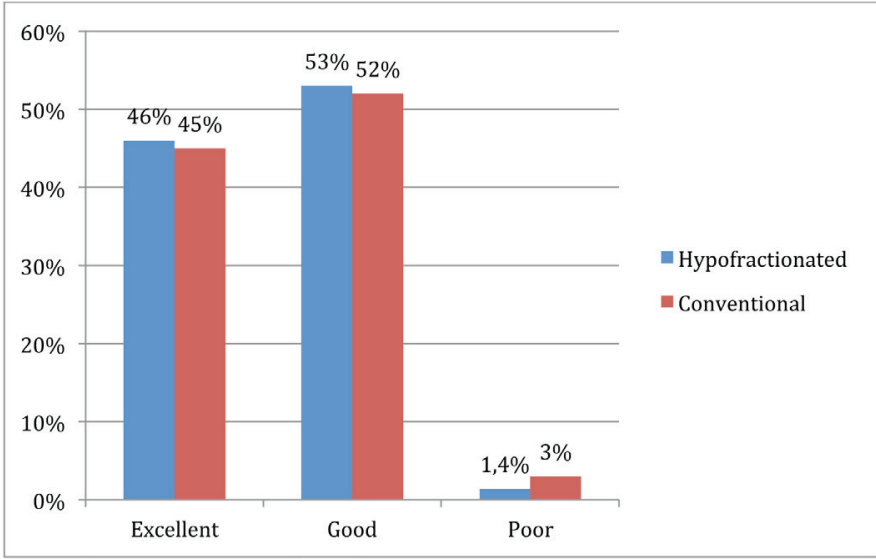

Figure 2. Cosmesis according to fractionation received.

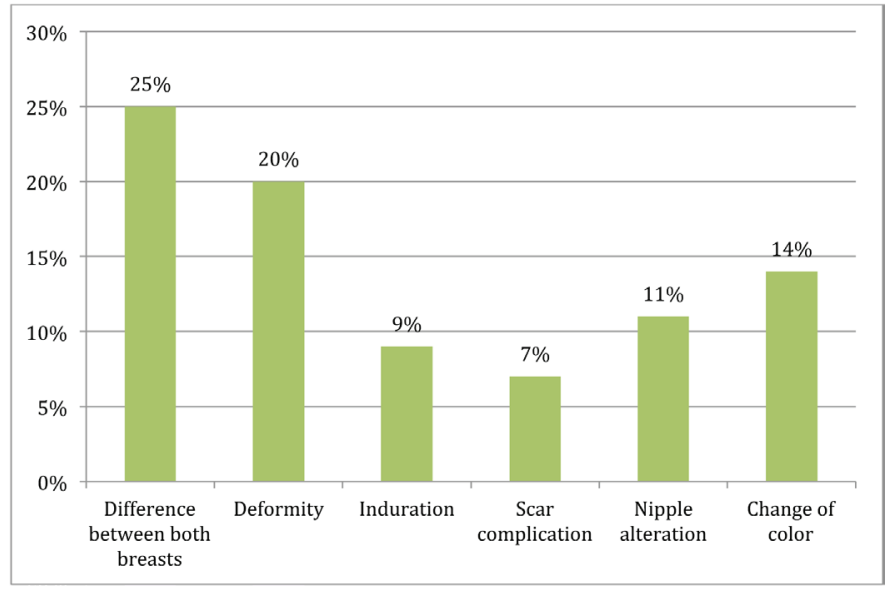

Figure 3. Presence of alteration affecting cosmesis. 


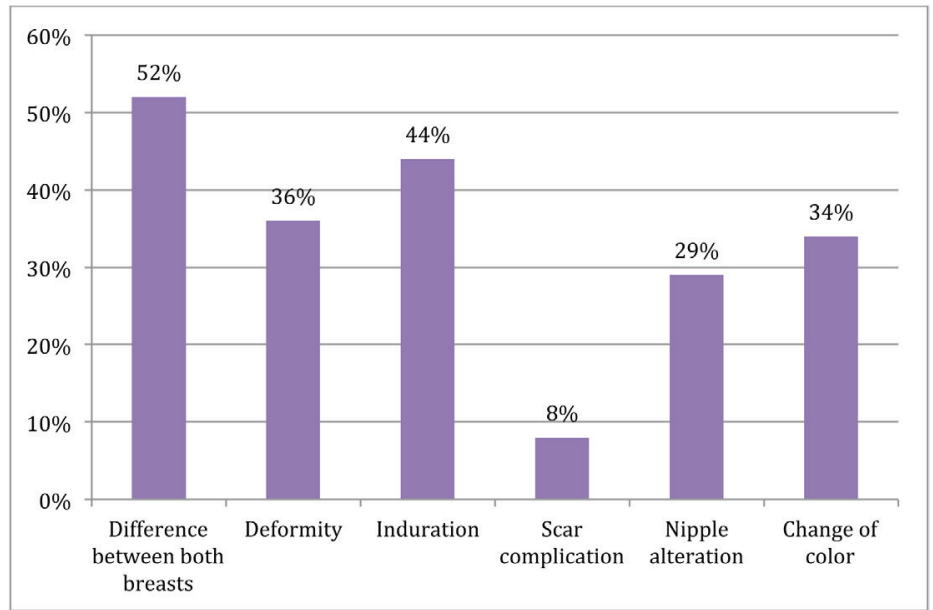

Figure 4. Presence of alteration does not affect cosmesis.

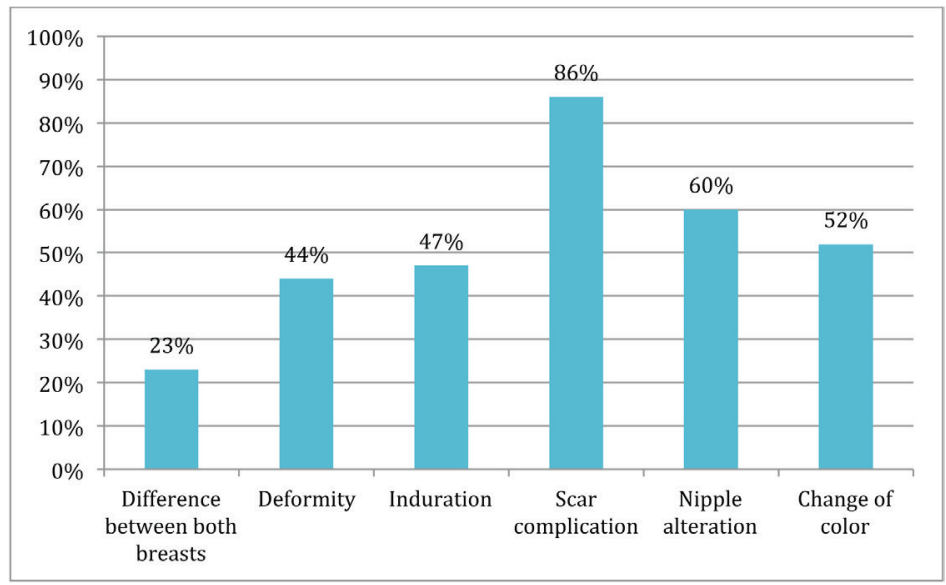

Figure 5. No alteration.

In a post-hoc analysis, $15 \%$ of patients younger than 58 years old reported breast colour change affecting cosmesis compared with $9 \%$ of patients older than 58 years old $(p=0.04)$. Patients under 58 years had a greater frequency of breast induration $(61 \%$ versus $49 \%$, $p=0.03)$.

Nine percent of patients with stages I-II breast cancer reported complications affecting breast cosmesis compared with $2 \%$ of patients with ductal carcinoma [ductal carcinoma in situ (DCIS)]. $(p=0.04) .14 \%$ of patients stage I-II referred colour change affecting cosmesis compared with $6 \%$ of those with DCIS $(p=0.03)$.

Pain was reported by $68 \%$ (190/279) of the patients. Most of them reported occasional pain $(62 \%, 172 / 279)$, whereas only $6 \%(18 / 279)$ reported permanent pain. The rest of the patients reported no presence of pain $(32 \%, 89 / 279)$. There were no statistically significant differences in the presence of pain between hypofractionated treatment and conventional treatment $(p=0.9)$.

When considering only the questionnaires before the start of RT and at the end of it, for the variable 'overall cosmesis', in both times the most frequent response was good cosmesis (54\% at baseline and $64 \%$ at discharge), while $3 \%$ and $4 \%$ reported poor cosmesis at the beginning and at discharge, respectively. 
Table 3. Pre-RT and at-discharge responses.

\begin{tabular}{|c|c|c|c|c|c|c|}
\hline & \multicolumn{3}{|c|}{ PRE RADIOTHERAPY } & \multicolumn{3}{|c|}{ AT DISCHARGE } \\
\hline & NO & $\begin{array}{c}\text { YES, IT DOESN'T } \\
\text { AFFECTS }\end{array}$ & YES, IT AFFECTS & NO & $\begin{array}{c}\text { YES, IT DOESN'T } \\
\text { AFFECTS }\end{array}$ & YES, IT AFFECTS \\
\hline DEFORMITY & $\begin{array}{l}58 \% \\
41 / 71\end{array}$ & $\begin{array}{l}27 \% \\
19 / 71\end{array}$ & $\begin{array}{l}15 \% \\
11 / 71\end{array}$ & $\begin{array}{c}40 \% \\
32 / 80\end{array}$ & $\begin{array}{c}36 \% \\
29 / 80\end{array}$ & $\begin{array}{l}24 \% \\
19 / 80\end{array}$ \\
\hline SCAR COMPLICATION & $\begin{array}{l}86 \% \\
60 / 70\end{array}$ & $\begin{array}{c}6 \% \\
4 / 70\end{array}$ & $\begin{array}{l}8 \% \\
6 / 70\end{array}$ & $\begin{array}{l}81 \% \\
65 / 80\end{array}$ & $\begin{array}{l}10 \% \\
8 / 80\end{array}$ & $\begin{array}{l}9 \% \\
7 / 80\end{array}$ \\
\hline NIPPLE ALTERATION & $\begin{array}{c}75 \% \\
53 / 71\end{array}$ & $\begin{array}{c}18 \% \\
13 / 71\end{array}$ & $\begin{array}{l}7 \% \\
5 / 71\end{array}$ & $\begin{array}{c}45 \% \\
36 / 80\end{array}$ & $\begin{array}{c}39 \% \\
31 / 80\end{array}$ & $\begin{array}{c}16 \% \\
13 / 80\end{array}$ \\
\hline
\end{tabular}

${ }^{*}$ Occasional pain

**Permanent pain

Both before the start of radiation therapy and at the end of it, the frequency described above is maintained, the alteration referred with greater frequency was 'difference between both breasts' (66\% before and $80 \%$ after RT), and in most, without affecting cosmesis. Colour change of the breast upon discharge was reported by $68 \%(54 / 80)$ of the patients and $26 \%(21 / 80)$ claimed that it affected their cosmesis. Thirty-three percent pointed out no change of normal breast colour after RT. Regarding pain, most patients reported occasional pain at the beginning and at the end of RT, no statistically significant difference was found between both time points. Thirty-three patients had questionnaire both before RT and at discharge (Table 3). Finally, 94\% of patients stated that they would accept treatment again.

\section{Discussion}

This study demonstrates that aesthetic result after conservative surgery and RT in patients with early-stage breast cancer is generally good as perceived by patients, given by $98 \%$ of them who refer excellent/good cosmesis, $46 \%$ excellent cosmesis, $52 \%$ good and only $2 \%$ poor results, which is comparable with data reported in previous literature where excellent or good assessment is described in 66\% [10], and in current studies where excellent or good cosmesis is reported in $70 \%$ and grade 3 or greater toxicity does not exceed $4 \%$ [2]. It has also been reported in other studies with prospective evaluation of cosmesis $93 \%$ good-excellent results and only $7 \%$ of unsatisfactory aesthetic outcome in patients treated with HF [11].

The alteration most frequently described was difference between both breasts, both globally and before the beginning and at discharge of RT. This is probably the most frequent alteration given the broad meaning that this question may have and it may also be valued differently by each patient. The percentage of patients who reported 'difference between both breasts' was 66\% before the start of RT, confirming what was mentioned above, sequelae of treatment begin with surgery and then remain or could be increased with adjuvant treatments.

The alteration 'change in normal breast colour' affected cosmesis in only a quarter of the patients at discharge, this could be due to management of patient expectations with respect to outcome and treatment toxicity, and that change in colour, either erythema and/or hyperpigmentation, is frequent and usually transient. 
There was no statistically significant difference in overall aesthetic result according to the fractionation used, consistent with that reported by other studies [2, 3]. As mentioned previously, studies that evaluated the use of a hypofractionated regimen versus a conventional schedule showed no inferiority in local control but, even more, toxicity was not increased in those patients receiving hypofractionated RT, even when the dose per fraction is greater [2-5]. No difference was found between overall evaluation of cosmesis between the two regimens, with $71 \%$ reporting good-excellent cosmesis in the CF group and $70 \%$ in HF up to 10 years of follow-up [2]. In other studies of HF, late toxicity was in favour of the hypofractionated regime, considering shrinkage, breast swelling and telangiectasia [3].

The randomized study by Shaitelman et al [7] presents data on toxicity between two RT schedules in patients with early-stage breast cancer, treated with conservative surgery and who were to receive RT only to the breast with a tumour bed boost in both groups. Most patients were overweight-obese and over $50 \%$ had the maximum dose (Dmax) of 107\% or higher relative to the prescribed dose. Acute toxicity was in favour of HF, with lower incidence of grade 2 or greater toxicity ( $78 \%$ in CF versus $47 \%$ in $\mathrm{HF}$ ), less fatigue (13\% versus $9 \%)$, pruritus $(74 \%$ versus $50 \%)$, breast pain $(65 \%$ versus $50 \%)$, dermatitis $(68 \%$ versus $36 \%)$ and hyperpigmentation $(20 \%$ versus $9 \%)$. At 6 months, there was no difference in toxicity between the two schedules, except in the presence of fatigue, which was higher in the CF group. Although the result of the assessments by the patients themselves is still pending, these data confirm the benefit of HF regarding breast toxicity.

In addition to this, a multicentre, observational study [12], which sought to compare toxicity between $\mathrm{HF}$ and $\mathrm{CF}$ according to physician and patient assessment, found higher frequency of grade 2 or greater toxicity during treatment as assessed by the treating physician $(67 \%$ in CF versus $32 \%$ in hypofractionated regimen; $p=0.003$ ), higher frequency of moderate or severe pain, grade 2 dermatitis, induration, chest wall pain, and moist and dry desquamation. According to the evaluation by the patients, there was greater frequency of pain, moist and dry desquamation, oedema and fatigue with CF [12]. This study represents a more familiar scenario, closer to routine clinical practice setting and closer to the cohort presented in our study, with comparable results to those obtained by us, where hypofractionated RT did not result in worse cosmetic outcome for our patients.

The choice between one fractionation schedule over another in our institution is based, beside disease and patient characteristics, on the capacity of obtaining the best dosimetric parameters possible. The use of modern techniques of 3D conformal RT has led to lower treatment toxicity [13]. In our analysis, most patients were treated with 3D conformal radiation therapy with CT planning, and in half of them field-in-field technique was used to homogenize dose. Boost using was not associated with worse cosmesis, even when higher frequency of fibrosis has been reported in patients receiving tumour bed boost [14]. However in other studies, while it is associated with acute and late skin toxicity, it is not described specifically as a risk factor for worse cosmesis [11]. The use of field-in-field technique was not associated with better cosmesis, probably because patients with worse anatomy required this technique (larger breasts, obese patients, etc.). It could be inferred that field-in-field could counteract the effects of a not ideal anatomy for radiation therapy.

Regarding pain, most patients had occasional pain, both before the start of RT and at the end of it, and the minimum percentage described constant pain. Analgesia requirements were not assessed. Dose inhomogeneity has been described as a predictor of pain after breast RT [15]. The dosimetry data were not collected in the first stage of the study, but it is possible to infer that-based on the good cosmetic results-the small variation in reported pain before and after RT, and the widespread use of field-in-field technique, a homogeneous dosimetry that could explain these results was achieved.

Patient age and tumour size are among the factors affecting cosmetic outcome [11]. In this study, it was found that patients younger than 58 years old reported higher frequency of breast induration, and presented more frequently with changes in the normal colour of the breast that affected cosmesis, while patients with stages I and II breast cancer more commonly referred to any change affecting cosmesis compared with those patients with DCIS. Although tumour size may partially explain these results, we may also consider the effect of surgery on the perception of cosmesis. The effect that axillary surgery had was not evaluated directly, but it may be that those with DCIS who do not have a sentinel node study, and therefore, have less surgical manipulation, have a better evaluation of breast cosmesis and toxicity.

One of the limitations of this study is the subjective nature of the questionnaire that is also not validated. This questionnaire was made based on other scales most widely used in the literature [2-10], although in most the evaluation is done by the physician or by a third-party observer, but not by the patient. In this study, neither a cosmesis analysis by the treating physician or by the third observer, nor objective measurements of aesthetic results such as photographs that allow to quantify changes described by patients were applied. Moreover, it is noteworthy that with photographs alone it is not possible to capture all the changes that can appear in the treated breast, such as induration or pain. Even when there is no objective analysis, it is no less valid to have the perception of our patients relative to the cosmetic outcome 
of their own treatment, because in addition to oncological outcome the quality of life of our patients cannot be a secondary issue. Finally, it is also possible that there is a selection bias by the treating physician, who could decide whether or not to apply the questionnaire.

\section{Conclusion}

In conclusion, this study found no difference between hypofractionated regimens compared with CF of radiation therapy in our patients in terms of cosmetic results, with no differences in the different variables measured with respect to fractionation received. Great satisfaction regarding the cosmetic outcome of cancer treatment has been reported, given by $98 \%$ of excellent/good cosmesis, and $94 \%$ of patients who would agree to receive the treatment again.

\section{References}

1. EBTCG (2011) Effect of radiotherapy after breast conserving surgery on 10 year recurrence and 15 year breast cancer death: meta-analysis of individual patient data for 10801 women in 17 randomised trials Lancet 378 1707-1716 https://doi.org/10.1016/ S0140-6736(11)61629-2

2. Whelan T, Pignol JP, and Levine M, et al (2010) Long-term results of hypofractionated radiation therapy for breast cancer $N$ Engl J Med 362 513-520 https://doi.org/10.1056/NEJMoa0906260 PMID: 20147717

3. Haviland J, Owen R, and Dewar J (2013) The UK standardisation of breast radiotherapy (START) trial of radiotherapy hypofractionation for treatment of early breast cancer: 10 year follow up results of two randomised controlled trials Lancet Oncol 14 1086-1094 https://doi.org/10.1016/S1470-2045(13)70386-3 PMID: 24055415

4. START Trialists' Group, Bentzen SM, and Agrawal RK, et al (2008) The UK standardisation of breast radiotherapy (START) trial A of radiotherapy hypofractionation for treatment of early breast cancer: a randomised trial Lancet Oncol $9331-341$ https://doi. org/10.1016/S1470-2045(08)70077-9 PMID: 18356109 PMCID: 2323709

5. START Trialists' Group, Bentzen SM, and Agrawal RK, et al (2008) The UK standardisation of breast radiotherapy (START) trial B of radiotherapy hypofractionation for treatment of early breast cancer: a randomised trial Lancet 371 1098-1107 https://doi. org/10.1016/S0140-6736(08)60348-7 PMID: 18355913 PMCID: 2277488

6. Taylor ME, Perez CA, and Halverson KJ (1995) Factors influencing cosmetic results after conservation therapy for breast cancer Int J Radiat Oncol Biol Phys 31 753-764 https://doi.org/10.1016/0360-3016(94)00480-3 PMID: 7860386

7. Shaitelman SF, Schlembach PJ, and Arzu I (2015) Acute and short-term toxic effects of conventionally fractionated vs hypofractionated whole-breast irradiation. A randomized clinical trial JAMA Oncol 1931-941 https://doi.org/10.1001/jamaoncol.2015.2666 PMID: 26247543 PMCID: $\underline{4635441}$

8. Hopwood P, Fletcher I, and Lee A (2001) A body image scale for use with cancer patients Eur J Cancer 37 189-197 https://doi. org/10.1016/S0959-8049(00)00353-1 PMID: 11166145

9. Racz J, Look Hong N, and Latosinsky S (2015) In search of a gold standard scoring system for the subjective evaluation of cosmetic outcomes following breast-conserving therapy Breast J 21 345-351 https://doi.org/10.1111/tbj.12423 PMID: 25940058

10. Harris J, Levene M, and Svensson G (1978) Analysis of cosmetic results following primary radiation therapy for stages I and II carcinoma of the breast Int J Radiat Oncology Biol Phys 5 257-261 https://doi.org/10.1016/0360-3016(79)90729-6

11. Ciammella P, Podgornii A, and Galeandro M (2014) Toxicity and cosmetic outcome of hypofractionated whole-breast radiotherapy: predictive clinical and dosimetric factors Radiat Onco/ 997 https://doi.org/10.1186/1748-717X-9-97 PMID: 24762173 PMCID: $\underline{4029983}$

12. Jagsi R, Griffith KA, and Boike TP (2015) Differences in the acute toxic effects of breast radiotherapy by fractionation schedule. Comparative analysis of physician-assessed and patient-reported outcomes in a large multicenter cohort JAMA Oncol 1 918-930 https://doi.org/10.1001/jamaoncol.2015.2590 PMID: 26247417 
13. Mukesh M, Barnett G, and Wilkinson J (2013) Randomized controlled trial of intensity modulated radiotherapy for early breast cancer: 5-year results confirm superior overall cosmesis J Clin Oncol 31 4488-4495 https://doi.org/10.1200/JCO.2013.49.7842 PMID: 24043742

14. Bartelink $\mathrm{H}$, Maingon $\mathrm{P}$, and Poortmans $\mathrm{P}$ (2015) Whole-breast irradiation with or without a boost for patients treated with breast-conserving surgery for early breast cancer: 20 year follow up of a randomised phase 3 trial Lancet Oncol 16 47-57 https://doi.org/10.1016/S1470-2045(14)71156-8

15. Mak KS, Chen YH, and Catalano PJ (2015) Dosimetric inhomogeneity predicts for long-term breast pain after breast-conserving therapy Int J Radiat Oncol Biol Phys 93 1087-1095 https://doi.org/10.1016/j.jirobp.2014.05.021 